\title{
Die Teilnovellen als letzter Akt der österreichischen Zivilgesetzgebung in Mitteleuropa*
}

\begin{abstract}
The Partial Amendments of the ABGB as the last Act of the Civil Law Legislation in Central Europe

In the early 1970s, Helmut Slapnicka published his book "Österreichs Recht ausserhalb Österreichs. Der Untergang des österreichischen Rechtsraums".** This volume constitutes an important and central starting point for further enquiries into the continued formal and substantial relevance of the realm of Austrian law. As a first step towards in-depths studies on the Austrian civil law area in the 20th century, this article presents a brief introduction to the last major act of Austrian civil law legislation and its continuing relevance in Central Europe.
\end{abstract}

Keywords: Austrian Civil Code 1811 - codifications - Teilnovellen 1914-16 - Joseph UNGER

\section{Zur Reform und Reform- bedürftigkeit des $\mathrm{ABGB}$ zu Beginn des 20. Jahrhunderts}

Das österreichische Allgemeine Bürgerliche Gesetzbuch aus 1811, das bis heute in großen Teilen unverändert in Geltung ist, wurde immer wieder wegen seiner Lebenskraft, ${ }^{1}$ seiner Vitalität und Elastizität gelobt. ${ }^{2}$ Doch gerade diese lange Geltungsdauer des österreichischen Zivilrechtskodex führte immer wieder zu Reformdebatten, ${ }^{3}$ die in manchen besonders sensiblen, ideologisch geprägten Bereichen wie dem Ehe-

\footnotetext{
* Dieser Beitrag ist eine kurze Vorstellung des kürzlich begonnenen Habilitationsprojekts der Autorin, das sich primär mit den Teilnovellen und deren Entstehung und Einfluss auf die Privatrechtsentwicklung in Österreich und Polen beschäftigt.

**SLAPNICKA, Österreichs Recht.

${ }^{1}$ KLEIN, Lebenskraft 32.

${ }^{2}$ OGRIS, Geschichte und Bedeutung 323.

${ }^{3}$ Unger, Entwicklungsgang 358f.; PFAFF, RANDA, STROHAL, Gutachten.
}

schließungs- und Ehescheidungsrecht über Jahrzehnte hinweg ohne Erfolg betrieben wurden. ${ }^{4}$ Insbesondere die Arbeiten am deutschen Bürgerlichen Gesetzbuch gaben den österreichischen Juristen den Anstoß, das ABGB kritisch auf Mängel hin zu untersuchen. ${ }^{5}$

Der entscheidende Impuls für den tatsächlichen Beginn der Reformarbeiten wird in der Literatur Joseph Unger und seiner 1904 veröffentlichten Schrift "Zur Revision des allgemeinen bürgerlichen Gesetzbuches" ${ }^{\prime 6}$ zugeschrieben.7 Wir dürfen annehmen, dass Ungers Aufsatz im Frühjahr 1904 erschien, den ersten Reaktionen folgend wohl Ende März 1904. In der Wochenschau der Juristischen Blätter, verfasst am 1. April 1904, wird bereits auf Ungers "soeben veröffentlich[te]" Schrift hingewiesen. ${ }^{8}$ Bereits vier Wo-

\footnotetext{
${ }^{4} \mathrm{Vgl}$. bspw. HARMAT, Ehe auf Widerruf 24-47.

${ }^{5}$ MITTEIS, Bürgerliche Gesetzgebung.

${ }^{6}$ UNGER, Revision.

${ }^{7}$ Vgl. bspw. DÖLEMEYER, Revision 275.

${ }^{8}$ ANONYMUS, Wochenschau 161.
} 
chen später am 29. April 1904 setzte der Kaiser auf Vorschlag des Ministerpräsidenten und Justizministers Ernest von Körber eine Kommission aus Theoretikern unter der Leitung von Joseph Unger ein. ${ }^{9}$ Die meisten Mitglieder rekrutierten sich aus dem Herrenhaus: $\mathrm{Zu}$ ihnen gehörte Emil Steinbach, zu diesem Zeitpunkt zweiter Präsident des Obersten Gerichtshofes, 10 Stanisław von Madeyski-Poray, wie auch Unger Mitglied des Reichsgerichts und ehemaliger Kultusminister sowie Antonín Randa, Universitätsprofessor an der tschechischen Universität in Prag [Praha]. (Noch) nicht im Herrenhaus vertreten waren die Mitglieder der Kommission Josef Schey, Universitätsprofessor an der Universität Wien, und Franz Klein, Sektionschef im Justizministerium, geistiger Vater der österreichischen Justizgesetze und nachmaliger Justizminister. Ein Blick auf den akademischen Bildungsweg der Kommissionsmitglieder, die allesamt Juristen waren, zeigt die Wienlastigkeit der Kommission: von sechs Mitgliedern hatten vier (Steinbach, Unger, Schey und Klein) in Wien studiert, ein Mitglied (Randa) in Prag und ein Mitglied (Madeyski) in Krakau [Kraków] und Lemberg [Lwiw].

Die Öffentlichkeit nahm reges Interesse an Ungers Reformkommission. Die Neue Freie Presse berichtete Ende April 1904 auf ihrer Titelseite von den Plänen der Regierung, ,eine Kodifikations-Kommission einzusetzen, an deren Spitze als Vorsitzender der Anreger der Idee, Reichsgerichtspräsident Dr. Unger, treten soll.“11 Abgerundet wurde dieser Bericht durch ein kurzes „Interview" mit Emil Steinbach. Zwar wollte sich Steinbach zu den Reformgebieten nicht

\footnotetext{
${ }^{9}$ DÖLEMEYER, Revision 275f. Vgl. auch zu den kurzen Biogrammen Ebd. 275-279.

10 Wiener Zeitung Nr. 103 v. 5. 5. 1904, 3. Die Angabe bei DÖLEMEYER, Revision 276 Anm. 11 ist irreführend. Steinbach wurde erst im September 1904 als erster Präsident beeidet. Wiener Abendpost (Beilage zur Wiener Zeitung) Nr. 208 v. 12. 9. 1904, 5.

${ }^{11}$ Neue Freie Presse Nr. 14250 v. 27. 4. 1904, 2.
}

festlegen, doch erklärte er, „daß die Schöpfung unseres bürgerlichen Rechtes in eine Zeit zurückdatiert, in der man von den kolossalen technischen Errungenschaften der Gegenwart, von der gänzlichen Umgestaltung des Produktionsprozess und des gesamten Wirtschaftslebens noch keine Ahnung haben konnte."12 Er ließ auch Reformen „auf dem Gebiet des Familienrechts“, insbesondere bei der Frage der Volljährigkeit, anklingen, sah „[d]iese und andere Fragen“ jedoch als "relativ nebensächlich gegenüber den schreienden Uebelständen, die dadurch entstanden sind, daß unser bürgerliches Recht in vielen Belangen den Anforderungen des modernen Wirtschaftslebens nicht mehr entspricht." 13

Anders ordnete Maria Schubert in ihrem Leitartikel in der Wiener Hausfrauen-Zeitung die Prioritäten bei der Revision des ABGB. Sie sah „eine Fülle von Hoffnungen [...] für zahlreiche Kreise“ geknüpft „an die Verwirklichung des geplanten Unternehmens. Von starker Bewegung ergriffen sind namentlich jene Klassen und Stände, welche von der Revision mit Sicherheit erwarten, daß dieselbe ihren gesellschaftlichen und wirtschaftlichen Bedürfnissen entgegenkomme, ihren Lebens- und Erwerbsverhältnissen günstigere Aussichten eröffne und sie von dem Drucke gewisser Bestimmungen des Gesetzbuches, die bisher schwer auf ihnen gelastet, befreien werde." 14 Klar war für Schubert, dass die Reformen „unmöglich an einem der jüngsten, aber nichtsdestoweniger hochwichtigen sozialen Probleme, an der Frage der Frauenemanzipation, vorübergehen [könne], ohne sich mit derselben, mindestens in einzelnen Bereichen, näher zu befassen. "15

Gemeinsam war Steinbach und Schubert die Forderung nach der Herabsetzung der Volljäh-

\footnotetext{
${ }^{12}$ Ebd.

${ }^{13}$ Ebd.

${ }^{14}$ SCHUBERT, Revision 277.

${ }^{15}$ Ebd.
} 
rigkeit. ${ }^{16}$ Als zentral sah Schubert die Verbesserung der Rechtsstellung der Frau, unter anderem durch die Öffnung der selbständigen Vormundschaft für Frauen und deren Zulassung als Zeugen von letzten Willenserklärungen. Ein Blick auf die Äußerungen von Emil Steinbach im Vergleich zu jenen von Maria Schubert zeigt deutlich die Diskrepanz der Erwartungen, die, determiniert durch die jeweiligen Lebensumstände, an die Reformen gesetzt wurden. In der österreichischen Tagespresse finden sich noch zahlreiche weitere Bemerkungen und Kommentare zu den Revisionsarbeiten am ABGB. Der umfangreiche Dialog nicht nur innerhalb der Juristenkreise, sondern auch in der Bevölkerung zeigte immer mehr reformbedürftige Bereiche auf, was sich in den Arbeiten der unterschiedlichen Kommissionen widerspiegelte und den Umfang der Novelle kontinuierlich wachsen ließ.

Für die Reform des ABGB sollte letztendlich die Unger'sche Kommission von untergeordneter Bedeutung sein. Noch im Sommer 1904 wurde parallel im Justizministerium mit der Erstellung einer Regierungsvorlage begonnen, diesmal jedoch unter Ausschluss der Öffentlichkeit. ${ }^{17}$ Querbeziehungen zu den Arbeiten der Reformkommission unter Unger lassen sich allerdings feststellen - 1907 beschäftigte sich die Reformkommission mit dem Entwurf des Justizministeriums kursorisch, auffallend sind auch personelle Verknüpfungen: Ab Jänner 1905 hatte Franz Klein die Leitung über das Justizministerium inne, ab Juni 1906 war er Justizminister. Die Arbeiten in Ungers Reformkommission stagnierten, zwar wurden einzelne Privatrechtsgebiete von ausgewählten Kommissionsmitgliedern ausgearbeitet - so bspw. das Erbrecht durch Unger und das Vertragsrecht durch Schey doch wurden diese kaum im Plenum beraten. ${ }^{18}$

\footnotetext{
${ }^{16} \S 21$ ABGB legte das Alter der Volljährigkeit mit dem 24. Lebensjahr fest.

${ }^{17}$ DÖLEMEYER, Revision 277f.

${ }^{18}$ Ebd. 278.
}

Die weiteren Reformschritte basierten auf der im Justizministerium ausgearbeiteten Regierungsvorlage, die im Dezember 1907 ins Herrenhaus eingebracht wurde. ${ }^{19}$ Im entsprechend eingerichteten Subkomitee finden sich bereits vertraute Namen: Stanisław von Madeyski-Poray und Franz Klein, aber auch der mittlerweile ins Herrenhaus ernannte Josef Schey waren wieder zur Arbeit am Revisionsentwurf berufen, hinzu kamen Karl Grünhut, Karl Grabmayr und Karl von Czyhlarz. Ein Blick auf den akademischen Hintergrund der Mitglieder zeigt, dass das Subkomitee wie auch schon die ursprüngliche Revisionskommission wienlastig war - drei (Schey, Klein, Grünhut) von sechs Mitgliedern hatten in Wien, ein Mitglied (Grabmayr) in Innsbruck, ein Mitglied (Czyhlarz) in Prag und ein Mitglied (Madeyski) in Krakau und Lemberg studiert. In ihren Beratungen berücksichtigte das Subkomitee das breitgefächerte Schrifttum zu den Revisionsvorschlägen. Während im Subkomitee manche cisleithanischen Universitäten nicht vertreten waren, spiegelt die juristische Literatur das Interesse der cisleithanischen Juristen wider - von Wien über Graz, Innsbruck, Prag, Krakau, Lemberg bis hin nach Czernowitz [Tscherniwzi]. ${ }^{20} \mathrm{Zu}$ bemerken ist dabei, dass die Reformdebatten nicht nur in deutscher Sprache geführt wurden, sondern sich beispielsweise auch polnischsprachige Juristenkreise vertieft mit der Problematik auseinandersetzten: Insbesondere ist der Lemberger Zivilrechtler Ernest Till zu nennen, der nicht nur in der deutschund polnischsprachigen Juristencommunity publizierte, ${ }^{21}$ sondern auch im Februar 1908 als Vortragender in der Wiener Juristischen Gesellschaft zum Thema „Die österreichische Zivilge-

\footnotetext{
${ }^{19}$ URBANITSCH, Honoratiorengremium 298.

${ }^{20}$ So bspw. der Grazer Professor HANAUSEK, Erbrecht. Eine Übersicht gibt DöLEMEYER, Teilnovellen 17911796.

${ }^{21}$ TILL, Rewizja Kodeksu; TILL, Revision.
} 
setznovelle" auftrat. ${ }^{22}$ Bereits die Regierungsvorlage aus dem Jahr 1907 hatte einen breiten Widerhall erhalten, die Debatte zog sich in den folgenden Jahren weiter, im Herrenhaus wurde die Regierungsvorlage stark überarbeitet und schlussendlich im Dezember 1912 vom Herrenhaus angenommen, zu einer inhaltlichen Auseinandersetzung mit dem Projekt durch das Abgeordnetenhaus kam es kriegsbedingt nicht mehr - der Reichsrat wurde am 25. Juli 1914 offiziell geschlossen. ${ }^{23}$

\section{Die Teilnovellen 1914-1916 - Gesetzgebung in der Krise}

Da der Reichsrat (bis 1917) nicht einberufen wurde, erließ der Kaiser die Änderungen zum ABGB, die sog. Teilnovellen, in Form von drei Notverordnungen, ${ }^{24}$ auch $\S 14$-Verordnungen genannt. $^{25}$ Somit erfolgten die ersten großen Novellierungen zum ABGB - insgesamt waren dabei mehr als 230 der 1502 Paragraphen betroffen - im Weg außerordentlicher Gesetzgebung. Dieser Umstand dürfte auch für die spätere Kritik an den Novellen mitverantwortlich sein, ${ }^{26}$ weniger betroffen waren davon die ersten beiden Teilnovellen. Wenige Wochen vor Erlassung der ersten Teilnovelle im Oktober 1914 erschien in den Juristischen Blättern ein Artikel des Reichsrastabgeordneten Julius Ofner unter dem Titel „Eine gerechtfertigte $\S 14$-Verordnung“. ${ }^{27}$ Darin verteidigte Ofner die Vorgehensweise mit dem Hinweis auf die Dringlichkeit der Materie. Die erste Teilnovelle umfasste das Personen-, Familien-, Erb- und Vormundschaftsrecht. Insbesondere wurde der rechtliche Handlungsrah-

\footnotetext{
${ }^{22}$ E., Wiener Juristische Gesellschaft 89.

23 ZIEGERHOFER, Hochblüte 260.

24 RGBl. 1914/276; RGBl. 1915/208; RGBl. 1916/69.

${ }^{25} \mathrm{Vgl}$. zu den $\S 14$-Verordnungen im Ersten Welt-

krieg: ZIEGERHOFER, Hochblüte.

${ }^{26}$ FUCHS, Teilnovellen 225.

${ }^{27}$ OFNER, § 14-Verordnung.
}

men von Frauen erweitert - sie durften nun zu Vormündern berufen werden und bei bestimmten Angelegenheiten als Zeuge fungieren. Die zweite Teilnovelle folgte im Juli 1915, änderte lediglich drei Bestimmungen des ABGB und brachte Neuerungen im Grenzberichtigungsrecht.

Anders sah es bei der dritten Teilnovelle aus, die den Rest des vom Herrenhaus abgesegneten Revisionsprojektes wieder mittels Notverordnung in Kraft setzte. Diese Teilnovelle war die umfangreichste von den dreien, sie konzentrierte sich primär auf sachen- und obligationsrechtliche Aspekte. Zwar handelte es sich allgemein bei den im Revisionsprojekt ausgearbeiteten Änderungen um Modernisierungen des ABGB, doch wurden die Bestimmungen der dritten Teilnovelle nicht als derart wichtig angesehen, als dass sie den Weg der außerordentlichen Gesetzgebung rechtfertigten. ${ }^{28}$ Hinzu kam, dass das Revisionsprojekt als solches lediglich im Herrenhaus, nicht jedoch in der Volkskammer, dem Abgeordnetenhaus, behandelt worden war, was noch zusätzlich für ein Zuwarten bis zur neuerlichen Einberufung des Reichsrates sprach. Schlussendlich erlebten die Teilnovellen keine inhaltliche Behandlung durch eine österreichische Volksvertretung. Versuche in der Zwischenkriegszeit, noch Änderungen in das ABGB einzufügen, scheiterten in Österreich abgesehen von kleinen Korrekturen.29

\section{Zum Fortleben des ABGB in den Rechtsordnungen der Nachfolgestaaten}

Nach dem Zusammenbruch der Monarchie entstanden auf deren Territorium Nationalstaaten. Charakteristisch für die Rechtsordnun-

\footnotetext{
${ }^{28}$ DÖLEMEYER, Teilnovellen 1790.

${ }^{29}$ Vgl. dazu StaUdigl-CiECHOWICZ, Zivilrecht 54-57.
} 
gen dieser Nachfolgestaaten ${ }^{30}$ Österreich-Ungarns war deren Rechtspartikularismus. Die neuen Staaten setzten sich aus Gebieten mit unterschiedlichen Rechtsordnungen zusammen, nach den Neugründungen wurden diese Rechtsordnungen bis auf das Verfassungsrecht zumeist provisorisch übernommen. ${ }^{31}$ Daraus resultierte eine große Rechtszersplitterung innerhalb der einzelnen Staaten. In Polen galten nebeneinander gar fünf unterschiedliche privatrechtliche Systeme, aufgeteilt nach dem räumlichen Geltungsbereich. ${ }^{32}$

Eine vergleichsweise geringe Rechtszersplitterung wies die Tschechoslowakei auf. Im tschechischen Teil blieb das ABGB in der Version nach den Teilnovellen gültig, im Rest der Tschechoslowakei galt das ungarische Recht. Das neu an die Tschechoslowakei vom Deutschen Reich angefallene sog. Hultschiner Ländchen wechselte 1920 vom BGB zum ABGB (und 1939 wieder zurück zum BGB). ${ }^{33}$

Ein besonders interessantes Bild zeichnen die Zivilrechtsordnungen des Königreichs der Serben, Kroaten und Slowenen. Hier begegnet uns das ABGB gleich in drei verschiedenen Formen. So war es inklusive der Teilnovellen in Dalmatien und Slowenien in Kraft, galt darüber hinaus - allerdings ohne Teilnovellen - in Kroatien-Slawonien und als subsidiäre Rechtsquelle in Bosnien-Herzegowina ${ }^{34}$. In den serbischen Gebieten hingegen war das Serbische Bürgerliche Gesetzbuch, das auf dem ABGB basierend 1844 eingeführt worden war, in Kraft. Primär ungarisches Recht galt in den zu Jugoslawien

\footnotetext{
${ }^{30}$ Der Begriff bezieht sich auf die territoriale nicht jedoch rechtliche Nachfolge nach der Doppelmonarchie.

31 SLAPNICKA, Österreichs Recht 11-14; für das Privatrecht vgl. BUSCH, BESENBÖCK, ABGB 581.

32 DZIADZIO, ABGB 205.

${ }^{33}$ BUSCH, BESENBÖCK, ABGB 587.

${ }^{34}$ Bosnien-Herzegowina war innerhalb des eigenen Territoriums von großer Diversität und Rechtspartikularismus geprägt. Vgl. DRAKIĆ, Codification 197.
}

gehörenden Teilen der Woywodina. In Montenegro hingegen war ein Gesetzbuch aus dem Jahr 1888 in Kraft. ${ }^{35}$

Auch in Rumänien war das Zivilrecht nach 1918 nicht einheitlich. Während ein Teil des Landes dem rumänischen Zivilgesetzbuch von 1864 unterlag, galt in den neu hinzugefügten Territorien - so vor allem in der Bukowina, Siebenbürgen, Banat und Bessarabien - noch das "alte“ Zivilrecht. In Teilen von Siebenbürgen und Banat war ungarisches Gewohnheitsrecht anwendbar, in der Bukowina und einem Teil Siebenbürgens das ABGB. Selbst innerhalb dieser ABGB-Gebiete gab es Unterschiede, in der Bukowina, die davor zu Cisleithanien gehört hatte, galt das ABGB mit den Teilnovellen, in Siebenbürgen hingegen ohne Teilnovellen. ${ }^{36}$

Selbst in Österreich gab es einen - wenn auch im Vergleich sehr kleinen - Bereich des Rechtspartikularismus im Zivilrecht mit dem ungarischen Eherecht im Burgenland. ${ }^{37}$

\section{Zur Erstreckung des Gel- tungsbereiches des ABGB nach dem Zerfall der Monarchie}

Trotz der staatsrechtlichen Umbrüche vergrößerte das $A B G B$ zunächst gar seinen Geltungsbereich. 1919 wurde das ABGB im Übermurgebiet, das nach dem Ersten Weltkrieg von Ungarn an Slowenien abgegeben werden musste, eingeführt. ${ }^{38} 1920$ erfolgte die Einführung des ABGB im bereits erwähnten sog. Hultschiner Ländchen. 1922 beschloss der polnische Ministerrat, das ABGB - wenn auch nur auf ein kleines Gebiet - zu erstrecken. ${ }^{39}$ Die Gebiete von Oberzips und Arwa, um die zwischen Polen

\footnotetext{
${ }^{35}$ DRAKIĆ, Codification 196.

36 So BusCH, BESENBÖCK, ABGB 593.

37 Vgl. dazu NeschwARA, Burgenland.

${ }^{38}$ BUSCH, BESENBÖCK, ABGB 593.

${ }^{39}$ DZIADZIO, ABGB 206.
} 
und der Tschechoslowakei ein Grenzkonflikt entstanden war, lagen im Einflussgebiet des ungarischen Rechts. 1920 kamen diese Gebiete an Polen. Zunächst wurde das ungarische Recht belassen und der Ministerrat ermächtigt, in diesen Gebieten im Verordnungsweg polnisches Recht einzuführen. Im September 1922 passte der Ministerrat der Ermächtigung folgend das Zivil-, Straf- und Prozessrecht an das in Krakau in Geltung stehende Recht an. ${ }^{40}$ Für das Zivilrecht bedeutete das die Übernahme des ABGB, ausgenommen waren davon explizit bestimmte erbrechtliche Bestimmungen. In Kraft blieb mit einigen Änderungen das ungarische Eherecht. Der Einführung des ABGB in diesen Gebieten lagen primär pragmatische Gedanken zugrunde - die benachbarten polnischen Gebiete unterlagen ebenfalls dem ABGB, es war auch einfacher, entsprechend ausgebildete Juristen $\mathrm{zu}$ finden, Krakau und die Jagiellonen Universität waren nur rund $100 \mathrm{~km}$ entfernt. ${ }^{41}$

Im gleichen Jahr erfuhr auch in Österreich das ABGB eine Erweiterung seines räumlichen Geltungsbereiches. Mit dem Staatsvertrag von St. Germain war ein Teil Westungarns, das deutschsprachige Burgenland, an Österreich gekommen. Ein eigenes Bundesverfassungsgesetz regelte die Fortgeltung des ungarischen Rechts, ermächtigte aber gleichzeitig die österreichische Bundesregierung, dieses unter bestimmten Umständen abzuändern bzw. aufzuheben. Als einer der zulässigen Gründe wurde die Rechtsänderung "aus Rücksichten der Rechtsangleichung“ genannt. ${ }^{42}$ Mit Mitte Juni 1922 wurde das ABGB im Burgenland in Kraft gesetzt. ${ }^{43}$ Einzig das ABGB-Eherecht wurde nicht rezipiert. ${ }^{44}$

\footnotetext{
${ }^{40}$ Dziennik Ustaw 1922/90 poz. 833.

${ }^{41}$ BUSCH, BESENBÖCK, ABGB 589.

$42 \S 6$ Abs. 2 BGBl. 1921/85.

${ }^{43}$ BGBl. 1922/315.

${ }^{44}$ BusCH, BESENBÖCK, ABGB 585f.; NESCHWARA, Burgenland; HARMAT, Ehe auf Widerruf 116-124.
}

\section{Das ABGB in den Kodifikationsarbeiten}

Die Rechtszersplitterung in den Zivilrechtsordnungen Mitteleuropas in der Zwischenkriegszeit führte zu einer starken Kodifikationsbewegung in diesem Bereich. Die Motive für die Schaffung eines neuen Gesetzbuches lagen einerseits in dem Bestreben nach einer einheitlichen Rechtsordnung und der Rechtssicherheit des Staatsvolkes. Dieser Grund alleine rechtfertigt jedoch nicht die Versuche, neue, eigene Gesetzbücher zu schaffen. Zeitsparender und leichter wäre die Erstreckung eines bereits geltenden Gesetzbuches auf das gesamte Staatsterritorium gewesen, wäre die Rechtseinheit der einzige Motor der Kodifikationsbewegung gewesen. Vielmehr spielten auch nationale und rechtspolitische Gedanken eine Rolle. Gerade in Polen wollten die Kodifikatoren keines der von den Teilungsmächten implementierten Gesetzbücher auf das gesamte Gebiet erstrecken. Einerseits galt es, das Gleichgewicht zwischen den ehemaligen Teilungsgebieten $\mathrm{zu}$ halten und nicht eines durch die Rechtsübernahme zu bevorzugen. Auf der anderen Seite verfolgte man die Idee, eine neue, eigene Kodifikation unter modernen Vorzeichen zu schaffen. ${ }^{45}$

Trotzdem scheint das ABGB in allen zivilrechtlichen Kodifikationsarbeiten der Nachfolgestaaten Österreich-Ungarns in der Zwischenkriegszeit auf. Während es in Polen primär ein Bestandteil der Vergleichsanalyse war und weniger eine Vorbildfunktion einnahm, nahmen die Zivilrechtsentwürfe der Tschechoslowakei und Jugoslawiens das ABGB für ihre eigene Kodifikation zur Grundlage. Ein Blick auf die Kodifikationsarbeiten in Polen zeigt, dass ABGB-Bestimmungen vereinzelt, also im Rahmen einer "selektiven Rezeption" 46 in die polnischen Gesetze und Entwürfe ihren Weg fanden.

\footnotetext{
${ }^{45}$ Malec, ABGB 243.

${ }^{46}$ DzIAdZIO, ABGB 219.
} 
Dieser Umstand ist auf verschiedene Ursachen zurückzuführen. Einerseits wurde das ABGB neben der "schweizerischen, französischen, spanischen, skandinavischen, serbischen, rumänischen und selbst japanischen ${ }^{47}$ Zivilgesetzgebung vergleichend analysiert. Mitbestimmend war wohl auch sein Ruf als eines der "besten Zivilgesetzbücher". Wichtig zu betonen erscheint hier auch die Frage des Einflusses der österreichischen Rechtskultur auf die Gesetzgebungsarbeiten im polnischen Staat. Gerade in den österreichischen Teilungsgebieten konnten sich durch die Etablierung zweier primär polnischsprachiger Universitäten eigene Zivilrechtsschulen bilden. ${ }^{48}$

\section{Der langsame Zusammen- bruch des österreichischen Zivilrechtsraumes}

Während das ABGB zu Beginn der Zwischenkriegszeit sogar - wie gezeigt wurde - an räumlichen Geltungsbereich gewinnen konnte, schrumpfte mit der fortschreitenden Kodifikationsarbeit in machen Staaten der sachliche Geltungsbereich. So trat in Polen 1934 das neue Obligationenrecht in Kraft, vereinheitlichte dieses Zivilrechtsgebiet für den Gesamtstaat und setzte folglich in diesem Bereich die Kodifikationen der Teilungsmächte außer Kraft. ${ }^{49}$ In Rumänien scheiterten die Kodifikationsarbeiten zum Zivilrecht, 1943 wurde schlussendlich das alte rumänische Zivilrecht, kodifiziert im rumänischen ZGB 1864, im gesamten Staat eingeführt, um (zumindest weitgehend) Rechtseinheit zu schaffen. ${ }^{50}$ Das endgültige formelle Ende für das ABGB in den Staaten Mitteleuropas kam mit der Etablierung der kommunistischen Regime.

\footnotetext{
${ }^{47}$ Ebd. 219.

${ }^{48}$ MALEC, ABGB 244-246.

${ }^{49}$ DZIADZIO, ABGB 212.

${ }^{50}$ ALUNARU, Rumänien 230.
}

Nach dem Ende des Zweiten Weltkrieges kamen Polen und die Tschechoslowakei in den sowjetischen Einflussbereich und adaptierten schrittweise ihre Rechtsordnungen dem sowjetischen Muster folgend. In Polen war das ABGB spätestens 1946 durch Einzelgesetze ersetzt worden, ein eigenes polnisches Zivilgesetzbuch folgte erst 1964. ${ }^{51}$ In der Tschechoslowakei büßte das ABGB 1950 mit der Einführung des tschechoslowakischen Bürgerlichen Gesetzbuchs fast den gesamten Geltungsbereich ein, lediglich die Bestimmungen zum Dienstvertrag waren bis 1964 in Kraft, ${ }^{52}$ so dass hier das Jahr 1964 das endgültige formelle Ende des ABGB darstellte.

\section{Korrespondenz:}

Dr. Kamila STAUdigl-CieCHOWICZ, LL.M. Universität Wien

Institut für Rechts- und Verfassungsgeschichte Schottenbastei 10-16

A - 1010 Wien

kamila.staudigl-ciechowicz@univie.ac.at ORCID Nr. 0000-0002-7018-535X

\footnotetext{
${ }^{51}$ BUSCH, BESENBÖCK, ABGB 590.

${ }^{52}$ Ebd. 588.
} 


\section{Abkürzungen:}

Siehe das allgemeine Abkürzungsverzeichnis: [http://www.rechtsgeschichte.at/media/abk.pdf]

\section{Literaturverzeichnis:}

Christian AlunARU, Rumänien: Ein nichtgeborenes Kind des rumänischen Zivilrechts in der Zwischenkriegszeit, in: Martin LÖHNIG, Stephan WAGNER (Hgg.), „Nichtgeborene Kinder des Liberalismus“? Zivilgesetzgebung im Mitteleuropa der Zwischenkriegszeit (Tübingen 2018) 227-272.

ANONYMUS, Wochenschau, in: Juristische Blätter 33 (1904) 160f.

Jürgen BusCH, Alexander BESENBÖCK, Von Mailand bis Czernowitz. Die Einführung des österreichischen ABGB, Gesamtstaatsidee und nationaler Partikularismus, in: Andreas BAUER, Karl H. L. WELKER (Hgg.), Europa und seine Regionen. 2000 Jahre Rechtsgeschichte (Köln 2007) 535-597.

Barbara DÖLEMEYER, Die Revision des ABGB durch die drei Teilnovellen von 1914, 1915 und 1916, in: Ius Commune 6 (1977) 274-303.

DIES., Die drei Teilnovellen zum ABGB (1914-1916), in: Helmut CoING (Hg.), Handbuch der Quellen und Literatur der neueren europäischen Privatrechtsgeschichte Bd. III/2 (München 1982) 17821799.

Gordana M. DRAKIĆ, Jugoslawien: Codification of Civil Law in the Yugoslav State between the two World Wars, in: Martin LÖHNIG, Stephan WAGNER (Hgg.), „Nichtgeborene Kinder des Liberalismus"? Zivilgesetzgebung im Mitteleuropa der Zwischenkriegszeit (Tübingen 2018) 195-225.

Andrzej DZIADZIO, Das ABGB in Polen, in: Elisabeth BERGER (Hg.), Österreichs Allgemeines Bürgerliches Gesetzbuch (ABGB). Eine europäische Privatrechtskodifikation Bd. III: Das ABGB außerhalb Österreichs (Berlin 2010) 203-226.

A. E., Wiener Juristische Gesellschaft, in: Juristische Blätter 37 (1908), 89f.

Gustav FuCHS, Zur Kritik der Teilnovellen, in: Allgemeine österreichische Gerichts-Zeitung 69 (1918) 225-227.

Gustav HANAUSEK, Das gesetzliche Erbrecht und Pflichtteilsrecht des Ehegatten in den Entwürfen einer Novelle zum allgemeinen bürgerlichen Gesetzbuche (Wien 1910).

Ulrike HARMAT, Ehe auf Widerruf? Der Konflikt um das Eherecht in Österreich 1918-1938 (= Studien zur Europäischen Rechtsgeschichte 121, Frankfurt aM 1999).

Franz KLEIN, Die Lebenskraft des allgemeinen bürgerlichen Gesetzbuches, in: Festschrift zur Jahrhundertfeier des Allgemeinen Bürgerlichen Gesetzbuches 1. Juni 1911 Bd. 1 (Wien 1911) 2-32.

Dorota MALEC, Das ABGB in den Arbeiten der polnischen Kodifizierungskommission 1919-1939. Ein positives oder negatives Modell?, in: Barbara DÖLEMEYER, Heinz MOHNHAUPT (Hgg.), 200 Jahre ABGB (1811-2011). Die österreichische Kodifikation im internationalen Kontext (Frankfurt a.M. 2012) 235-253.

Ludwig MitTEIS, Die bürgerliche Gesetzgebung für das deutsche Reich, in: Neue Freie Presse Nr. 11600 v. 8. 12. 1896, 2f.

Christian NESCHWARA, Überleitung ungarischen Rechts im österreichischen Burgenland nach 1921, in: J. NECKÁř, M. RADVAN, D. SEHNÁLEK, J. VALDHANS (Hgg), Dny práva - 2008 - Days of Law (=Acta Universitatis Brunensis Iuridica 337, Brünn 2009) 469-495.

Julius OFNER, Eine gerechtfertigte $\S 14$-Verordnung, in: Juristische Blätter 43 (1914) 389.

Werner OGRIS, Zur Geschichte und Bedeutung des österreichischen Allgemeinen bürgerlichen Gesetzbuches (ABGB) [1989], in: Werner OGRIS, Elemente europäischer Rechtskultur, hg. v. Thomas OLECHOWSKI (Wien-Köln-Weimar 2003) 311-331.

Leopold PfafF, Antonín RANDA, Emil StROHAL, Drei Gutachten über die beantragte Revision des 30 . Hauptstückes im II. Theile des a.b. Gesetzbuches (Wien 1880).

Maria SCHUBERT, Die Revision des österreichischen bürgerlichen Gesetzbuches und die Frauen, in: Wiener Hausfrauen-Zeitung Nr. 28 v. 10. 7. 1904, $277 f$.

Helmut SLAPNICKA, Österreichs Recht ausserhalb Österreichs. Der Untergang des österreichischen Rechtsraums (= Schriftenreihe des österreichischen Ost- und Südosteuropa-Instituts 4, Wien 1973).

Kamila STAUDIGL-CiechOWICZ, Österreich: Das österreichische Zivilrecht und die österreichische Zivilrechtswissenschaft in der Zwischenkriegszeit, in: Martin LÖHNIG, Stephan WAGNER (Hgg.), „Nichtgeborene Kinder des Liberalismus“? Zivilgesetzgebung im Mitteleuropa der Zwischenkriegszeit (Tübingen 2018) 39-69.

Ernest TILL, Czy rewizja Kodeksu cywilnego konieczna?, in: Przegląd Prawa i Administracji 31 (1906) 601-614. 
DERS., Revision oder Ergänzung des allgemeinen bürgerlichen Gesetzbuches?, in: Allgemeine österreichische Gerichts-Zeitung 58 (1908) 41-45.

Joseph UNGER, Ueber den Entwicklungsgang der österreichischen Civiljurisprudenz seit der Einführung des allgemeinen bürgerlichen Gesetzbuchs, in: Jahrbücher der deutschen Rechtswissenschaft und Gesetzgebung 1 (1855) 353-359.

DERS., Zur Revision des allgemeinen bürgerlichen Gesetzbuches. Eine legislativpolitische Studie, in: Zeitschrift für das Privat- und Öffentliche Recht der Gegenwart 31 (1904) 389-406.
Peter URBANITSCH, Das elitäre Honoratiorengremium und die Vertiefung der civil society: Das österreichische Herrenhaus und die Revision des ABGB vor dem Ersten Weltkrieg, in: Wilhelm BRAUnEDER, Milan HLAVAČKa (Hgg.), Bürgerliche Gesellschaft auf dem Papier: Konstruktion, Kodifikation und Realisation der Zivilgesellschaft in der Habsburgermonarchie (=Schriften zur Europäische Rechts- und Verfassungsgeschichte 59, Berlin 2014) 295-309.

Anita ZIEGERHOFER, Die zweite Hochblüte des § 14, in: BRGÖ 8 (2018) 259-273. 